\title{
Short telomeres in gout linked with flares and CVD
}

...frequency of
gouty flares
was strongly
associated
with shorter
telomere
length

Telomere length, which is commonly used as a marker of cellular senescence, is decreased in patients with gout as compared with healthy individuals, according to a study led by researchers at the Radstake lab at UMC Utrecht. The same study also found that shorter telomeres are associated with hallmarks of gout and risk of cardiovascular disease.

"In the last decade it has become clear that chronic inflammation leads to cardio-
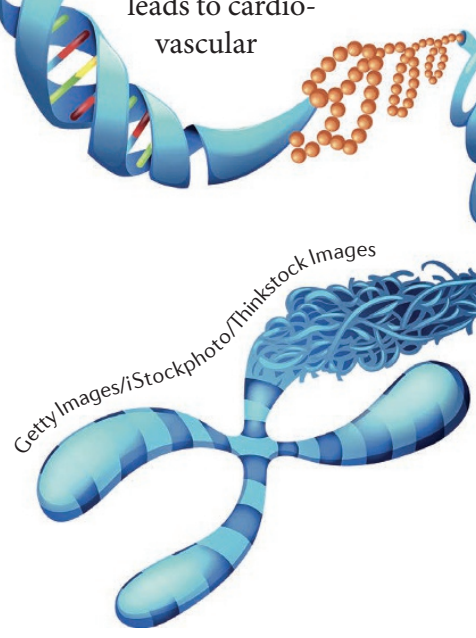

disease and increased immune cell turnover," explains Jasper Broen, corresponding author of the study. Broen and colleagues had previously shown that patients with gout and cardiovascular disease are in a state of chronic inflammation. "This finding led us to hypothesize that this chronic inflammation in gout leads to an increased rate of cellular senescence, which in itself is a contributor to the prevalence of cardiovascular disease," says Broen.

In the latest study, telomere length of peripheral blood mononuclear cells was significantly shorter in patients with gout $(n=145)$ than in healthy individuals $(n=273)$ in a Dutch cohort. This association was also observed in a sepa-

alone. Notably, frequency of gouty flares was strongly associated with shorter telomere length.

"We believe this shorter telomere length in patients with gout is due to increased leukocyte turnover as a consequence of inflammation," Broen says. "Our finding that patients with the most flares, and hence the most inflammatory episodes, have the shortest telomeres further substantiates this hypothesis. In turn, the patients with the shortest telomeres in our cohort have the highest burden of cardiovascular disease, which might reflect a previously described link between senescence and cardiovascular disease," adds Broen.

The authors suggest that large prospective studies are needed to explore whether intensive treatment of gout could reduce cellular senescence and thereby modify the risk of cardiovascular disease.

Sarah Onuora gout and 293 healthy individuals. Gout and cardiovascular disease were both independently associated with shorter telomere length, and telomeres were shorter in patients with both gout and cardiovascular disease than in patients with either condition
ORIGINAL ARTICLE Vazirpanah, N. et al. Patients with gout have short telomeres compared with with flare frequency and cardiovascular disease in gout.Ann. Rheum. Dis. http://dx.doi.org/10.1136/ annrheumdis-2016-210538 (2017) healthy participants: association of telomere length 